\title{
Research on customization and recommendation based personalized information services system for mobile learning
}

\author{
Yongmei Zhang ${ }^{1}$ Kejun Zhuang ${ }^{2}$ \\ ${ }^{1}$ Network Center, China West Normal University \\ ${ }^{2}$ Experiment Center, China West Normal University
}

\begin{abstract}
Personalized information service is necessary for learners groping in the ocean of mobile learning information. Currently customization system and recommendation system can not independently and perfectly achieve real intelligent and personalized push to meet the requirements of personalized information services. Firstly, based on the summary of the mobile learning information service mode, its development trend is analyzed, and it concludes that it needs combine customization and recommendation for mobile learning information service to get real personalized learning services. Then, 3 layers framework: the data layer, the business logic layer, and the application layer for the customization and recommendation based personalized information services system is described. Finally, the process of system and key technology of achievement are discussed.
\end{abstract}

Keywords: Mobile Learning; Personalization Information Service; Customization System; Recommendation System; Model of Student

\section{Introduction}

People makes full use of the time called garbage time, such as in the bus, train, taxi, traffic jams on the road to play webgames, surf the web or learning on the mobile phone. For providing users with more effectively personalized information push service, the server must collect a large number of user's private information. But it has incurred criticism from privacy advocates.

There are two areas in the existing research. One is the study of customization-push system, and the other is the study of the personalized recommendation system. Two areas are mutually isolated, and are not integrated. It causes that a customization system can not achieve real intelligent push, and a recommendation system can not realize personalized push. So the deep-seated personalized push services still need customization to get the user's behavior and private information, in order to overcome blindness and enhance pertinence. In the mobile learning process it also needs combine customization and recommendation for mode of mobile learning information service to get real personalized learning services.

\section{Mode of Information Services for Mobile Learning}

Mobile information service is to provide information service for mobile users via mobile networks and hand-held mobile 
devices [1]. Mode of information services for mobile learning is a way that information provider (server) interactive with learner's terminal.

\subsection{SMS Based Information Service Mode}

The user sends the short message via the mobile terminal to the teaching server in the Internet. The teaching server analyzes the user's short message, convert into request data, process them, and then send to user back. [2][3]

\subsection{Browse and Download-based Information Service Mode}

By the mode, learners can browse, inquire and download all kinds of the course resources on the WAP server or Web server, at any time and any where, to accomplish some learning activity. [4]

\subsection{E-mail Based Information Service Mode}

The multimedia mail service is a kind of non-real time service based on the mobile communication network and technology of the data-bearing. That business allows users sending multimedia messages between the mobile terminal and the Internet. [5]

\subsection{VOD Based Information Service Mode}

Compared with the VOD of the traditional Network Education, it has greater flexibility, more suitable for the crowd in the modern fast-paced learners, and it will bring huge impact on the traditional Distance Education, and the Television Education.[6]

\section{Development Tendency of the In- formation Service Mode for Mobile Learning}

Currently there are two categories of modes, active service and passive service.
SMS(Short Message Service) based information service mode, browse and download based information service mode, E-mail based information service mode and VOD(Video-On-Demand) based information service mode all have the features of the passive services.

There is another active information service- - active recommendation mode. In this mode, learners customize their own resources, and the server pushes information according to their customization. This active service mode allows learners to timely access to information, but incurred a lot of criticism. The main reason is such lack of pertinence for the information recommended that it can not meet the real interests and needs of the learners. And another reason is that the server collects so much private information, for more effectively push service, that has incurred criticism from privacy advocates.

We believe that push information, combining recommendation and customization, could improve the accuracy, and enhance learning efficiency of personalized information services.

\section{Framework Construction of the Personalized Information Services System for Mobile Learning}

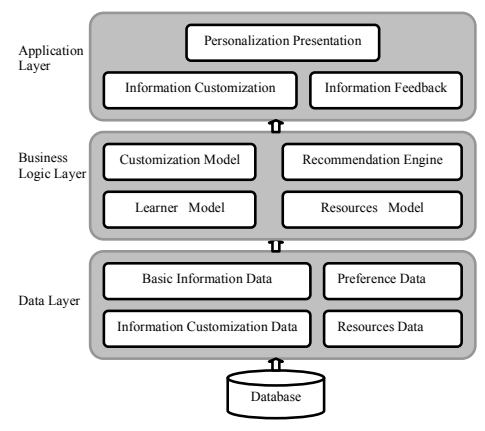

Fig. 1: Framework construction of the personalized information services system for mobile learning 


\subsection{Application Layer}

Application layer provides mobile learners with personalized interface of learning customization, browsing, learning and evaluation. Learners need to evaluate the contents recommended, and the system collects this kind of the feedback to improve the system mode.

\subsection{Business Logic Layer}

Main function is that the system builds learner interest model according to the learner's preference mode, and builds learners customization model. Two models provide conditions to recommendation engine. At the same time system builds resources model according to the learner interest model.

\subsection{Data Layer}

The layer provides business logic layer with various categories of data. It mainly includes: learner basic information data, learner preference data, information customization data, and learning resources data. They would be used to match the resources with the personalized information recommendation.

\section{Process of the Customization and Recommendation Based Persona- lized Information Services system for Mobile Learning}

\subsection{Customization of the Interactive Interface}

Personalized information services provide user with interface customization services. The services allow learners to choose interface display, including interface structure customization, and interface contents customization and interface update customization.

\subsection{Learning Contents and Resources Customization}

Learning contents and resources customi- zation tailor the required specific learning resources based on the learner's specific characteristics and demand. These learning resources include: (1) internal resources, learning resources that the system has. (2) Network resources (internet learning resources).

\subsection{Building Learner Interest Model}

Because of the each learner's different experience and background, there is a big difference in learners learning content, learning progress, learning styles and learning strategies. In order to effectively implement the personalized recommendation service and make it more targeted, it is necessary to analyze the learner's learning preferences model. The data mining technology is adopted to acquire the learners' preferences mode. The mined data includes the learner's log data, adaptability of learning content and learning difficulty, user behavior records, user transaction history, user collection, user registration information, personal evaluation and feedback information.

\subsection{Building Learners Customization Model}

Because of the different abilities of each learner, there is a great difference in learners' learning plan and time management, contents and progress. The system needs to push learning information depending on customization information of learners.

\subsection{Matching Resources Needs Retrieval Mode Library and Resources}

Retrieval mode library: preference mode library stores different preference patterns of learners, the learning progress, learning interest and learning strategies. The role of the resource recommendation engine is that utilizing content-based filtering and collaborative filtering recommendation algorithm to cluster 
learners with similar preference patterns, and predicting learner interest or resource preferences based on the neighborslearner's resource preferences and evaluation.

\subsection{Personalized Learning Resources Presentation}

The presentation of personalized resource is an output process of the result of recommendation. The engine also needs to analyze the characteristics of learning resources and content, and learner customization information. Based on the preferences of learners recommending resources, the engine improves the efficiency and effectiveness of the personalized recommendation. After analysis of customized information and recommended information, the system presents the information automatically to learner.

\section{The Key Technology For Achieve- ment}

\subsection{Social Tagging}

The practice of generating electronic tags or keywords by users rather than specialists as a way to classify and describe online content: Social tagging can enhance students' access to online collections of art. Compared with the traditional classification, the social tagging has the following characteristics or advantages: classification is booked generally in advance. It means that the category of the resources has been ordained in advance. But the social tagging is different. The tagging is added after the resource is completed. Social tagging in the environment of personalized mobile learning can facilitate clustering learning resources and information, matching resources and customized information, and improving the accuracy of the recommendation.

\subsection{Personalized Recommendation Technology}

Typically, personalized recommendation uses rule filtering, content filtering and collaborative filtering approach. Personalized recommendation algorithm includes association rules, cluster analysis, sequential pattern mining and regression analysis. Collaborative filtering technology was first developed in Tapestry system, and was mainly used to solve the problem of the screening of the e-mail. Collaborative filtering algorithm can find and recommend learners' potential needs and novel learning resources, and implement recommendation. The specific algorithms have been discussed in a lot of literatures, and don't repeat here.

\section{References}

[1] MAO Yi-hong, "On the Content and Mode of Mobile Information Service, "Information Science, pp. 210215,2012(2).

[2] Wang Xiaodong, Li Yanmin, "Mobile phone SMS based mobile learning, "China Educational Technology, pp. 114-117,2007 (1).

[3] Sun Yaoting, "Practice and research on the M-Learning and mobile services, "Distance Education in China, pp. 68-70,2008 (8).

[4] Fu Hui, "Design and Achievement of the mobile learning system, "Thesis of the master degree. Wuhan: Central China Normal University,2008.

[5] http:// www.linezine.com/ 2.1/features/cqmmwiyp.htm.

[6] Zhu Shouye, "Mobile learningoriented curriculum design and learning mode, "China Educational Technology, pp,67-70,2008(12). 